\title{
TRACKING MICRORESONATOR Q-FACTOR IN CLOSED-LOOP OPERATION
}

\author{
J.H. Seo, K.S. Demirci, S. Truax, L.A. Beardslee and O. Brand
}

School of Electrical and Computer Engineering, Georgia Institute of Technology, Atlanta, Georgia, USA

\begin{abstract}
A method to extract and track the quality factor (Q-factor) of microresonators in closed-loop operation is presented. The proposed Q-factor tracking approach relies on a controlled stiffness modulation of the resonator introduced by a second feedback loop and, thus, solely requires frequency measurements. Furthermore, the proposed concept is applicable to all resonant microstructures featuring excitation and detection elements. The feasibility of the proposed method is verified experimentally by tracking the Q-factor variations of a micromachined resonator caused by ambient temperature changes.
\end{abstract}

\section{INTRODUCTION}

In resonant sensors utilizing a frequency output, the Q-factor is one of the key parameters, which affects the (short-term) frequency stability and, thus, the sensor resolution. The Q-factor is related to the mechanical properties of the resonator, namely its stiffness, mass, and damping. Any property variation of the microresonator in response to a measurand results in a quality factor change. Therefore, the Q-factor can be used to investigate the response of a resonant sensor to environmental changes. This fact has been exploited by using the Q-factor to sense e.g. temperature [1, 2], fluidic properties such as viscosity and density [1, 3, 4], and pressure [5]. By measuring both resonance frequency and quality factor changes, simultaneous sensing of multiple measurands becomes possible.

The Q-factor change of a resonator is generally detected either by measuring its oscillation amplitude [2] or its open-loop transfer characteristic [4]. Approaches based on an amplitude measurement require precise $\mathrm{A} / \mathrm{D}$ conversion, in which case the advantages of a resonant sensor are not fully utilized. Methods based on the open-loop transfer characteristic of the resonator typically require expensive equipment, such as a network analyzer, and continuous in-situ monitoring of $\mathrm{Q}$ during normal sensor operation is not feasible.

In this work, we propose a new Q-factor extraction and tracking method in closed-loop operation based on a controlled stiffness change of the resonator, relying solely on frequency data. Thereby, the Q-factor is measured along with the measurandinduced frequency shift during normal sensor operation. This simultaneous operation allows realization of multi-functional resonant sensing systems, in which environmental (e.g. temperature) changes as well as the main measurand of a resonant sensor are detected at the same time without need for additional sensing devices.

\section{PRINCIPLE OF Q-FACTOR TRACKING}

In a sensing application, the microresonator is generally incorporated in an amplifying feedback loop as the frequencydetermining element, and the measurand can simply be detected by measuring the resonance frequency change using a frequency counter. To sustain the harmonic oscillation of a microresonator at a specific resonance mode, the excitation force generated by the feedback loop is phase matched with the vibration velocity of the resonator to compensate for the damping force (see main loop in Fig. 1). This way, the effective damping coefficient of the overall closed loop system becomes zero and a self-sustaining oscillation can be initiated and maintained.

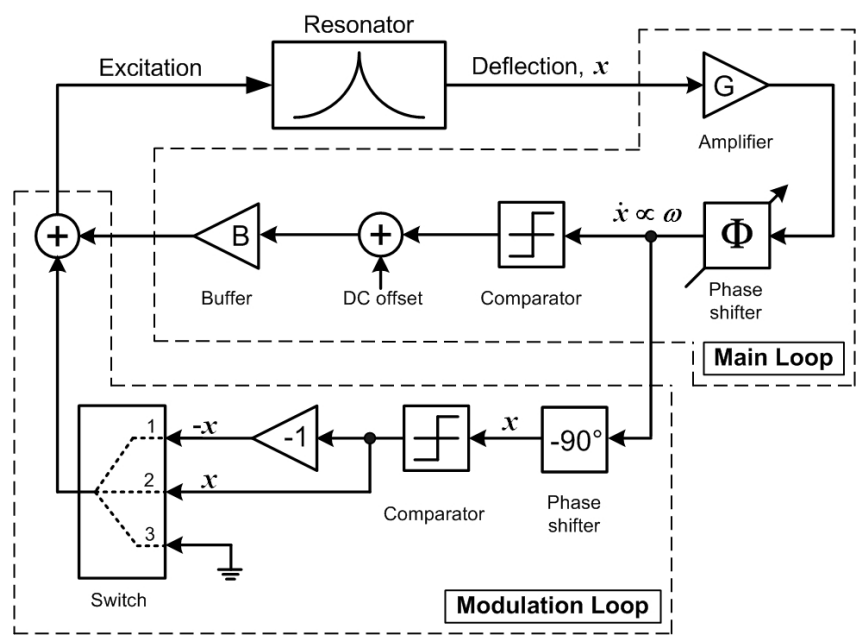

Figure 1: Schematic diagram of feedback circuitry with main loop and modulation loop for the proposed Q-factor tracking.

If an excitation force having the same phase as the resonator deflection signal is generated by a secondary electronic feedback loop (see modulation loop in Fig. 1) and applied to the resonator in addition to the force generated by the main loop, the effective stiffness of the resonator can be modulated by the additional feedback loop [6]. Depending on the direction of the applied force with respect to the resonator deflection, the effective stiffness of the resonator can be either reduced or increased. As a result, the resonance frequency of the harmonic oscillator is changed in response to the modulated effective stiffness.

This effective stiffness modulation method has been applied to extract relative stiffness changes of a microresonator for temperature compensation [7]. To extract the relative stiffness change, the additional feedback loop was composed of a phase shifter and a linear amplifier. A more detailed description of the above mentioned method can be found in [8].

If the linear amplifier of the modulation loop is replaced by a comparator, relative Q-factor changes $\Delta Q / Q$ can be extracted by analyzing the frequency changes caused by the additional feedback loop. Using the describing function of a comparator, defined as the complex ratio of the fundamental harmonic component of the comparator output signal to its input sinusoidal signal [9], the motion of a resonator with enabled modulation loop can be described by Eq. (1). Thereby, the microresonator is approximated by a simple second-order system consisting of mass $m$, damper $b$ and spring $k$.

$$
m \ddot{x}+b \dot{x}+k x=\psi_{\text {main }} \frac{\dot{x}}{|\dot{x}|}+\psi_{\text {mod }} \frac{x}{|x|}
$$

The force amplitudes $\psi_{\text {main }}$ and $\psi_{\text {mod }}$ in Eq. (1) are defined as: 


$$
\psi_{\text {main }}=\chi \gamma \frac{4 M_{\text {main }}}{\pi}, \quad \psi_{\text {mod }}=\chi \gamma \frac{4 M_{\text {mod }}}{\pi}
$$

Here, $\chi$ is the transfer function of the generated mechanical force to an applied electrical excitation signal, $\gamma$ is the transfer function of the electrical read-out signal to the resonator deflection, and $M_{\text {main }}$ and $M_{\text {mod }}$ are the amplitudes of the square wave signals generated by the main loop and modulation loop, respectively. By combining Eqs. (1) and (2), the system equation for the resonator shown in Fig. 1 is expressed by:

$$
m \ddot{x}+\left[b-\frac{\psi_{\text {main }}}{|\dot{x}|}\right] \dot{x}+\left[k-\frac{\psi_{\text {mod }}}{|x|}\right] x=0
$$

Considering that the force generated by the main feedback loop is the same as the damping force to sustain a harmonic oscillation at the mechanical resonance frequency, the magnitude of the steady-state vibration velocity $\dot{x}$ and the vibration amplitude $x$ of the resonator embedded in the feedback circuit are obtained from Eq. (3) by forcing the effective damping force (second term in Eq. (3)) to zero, i.e. $|\dot{x}|=\psi_{\text {main }} b^{-1}$ and $|x|=\psi_{\text {main }} b^{-1} \omega^{-1}$ (with $|\dot{x}|=\omega|x|$ ). Thus, the equation of motion becomes:

$$
m \ddot{x}+\left[k-\frac{\psi_{\text {mod }}}{\psi_{\text {main }}} b \omega\right] x=0
$$

Using the relation $Q=k b^{-1} \omega^{-1}$, Eq. (4) can be expressed by:

$$
m \ddot{x}+\left[k\left(1-\frac{\psi_{\text {mod }}}{\psi_{\text {main }}} \frac{1}{Q}\right)\right] x=0
$$

If the amplitudes of the excitation signals generated by the main and modulation loops are equal, i.e. $\psi_{\text {main }}=\psi_{\text {mod }}$, Eq. (5) is simplified to:

$$
m \ddot{x}+\left[k\left(1-\frac{1}{Q}\right)\right] x=0
$$

Eq. (6) shows that the relative stiffness change induced by the modulation loop is related to the Q-factor of the resonator. In fact, the stiffness is modified by a factor of $\left(1-Q^{-1}\right)$ by the modulation loop in Fig. 1.

Depending on the polarity of the signal in the modulation loop, either positive or negative effective stiffness changes can be produced resulting in an increase or decrease of the resonance frequency. Assuming that the additional spring force caused by the modulation loop has a spring constant of $C$, the resonance frequencies with positive and negative stiffness modulation, respectively, become:

$$
\omega_{\text {pos }}=\sqrt{\frac{k+C}{m}}, \quad \omega_{\text {neg }}=\sqrt{\frac{k-C}{m}}
$$

From the ratio of the frequencies $\omega_{\text {pos }} / \omega_{\text {neg }}$, the Q-factor of the resonator can be calculated:

$$
Q=\frac{\alpha+1}{\alpha-1}, \quad \alpha=\left(\frac{\omega_{\text {pos }}}{\omega_{\text {neg }}}\right)^{2}
$$

Therefore, the Q-factor (or the relative Q-factor change) of a microresonator can be extracted and tracked by measuring the resonance frequency changes caused by the periodically enabled modulation loop of Fig. 1.

It should be noted that Eqs. (1)-(9) only hold when the excitation signals generated by main and modulation loop are exactly phase matched with the vibration velocity $\dot{x}$ and the deflection of the resonator $x$, respectively. In a real system, the phase-tuning is done by an electronic circuit, such as an all-pass filter, and there is a possibility of a tuning error, which will introduce an error in the estimated Q-factor. However, the error caused by the phase tuning can be minimized if both the positive and the negative frequency changes induced by the modulation loop are used in extracting the relative Q-factor change of the resonator.

\section{EXPERIMENTAL SETUP}

To verify the feasibility of the proposed Q-factor tracking method, the experimental setup shown in Fig. 2 has been implemented.

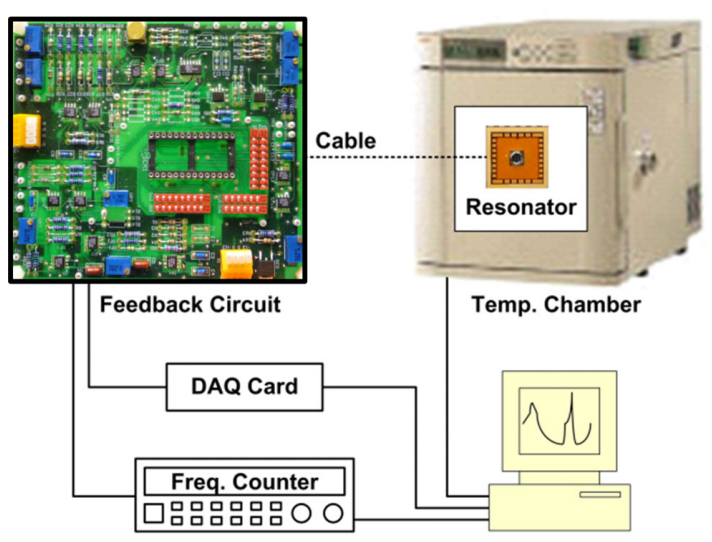

Figure 2: Schematic diagram of the experimental setup.

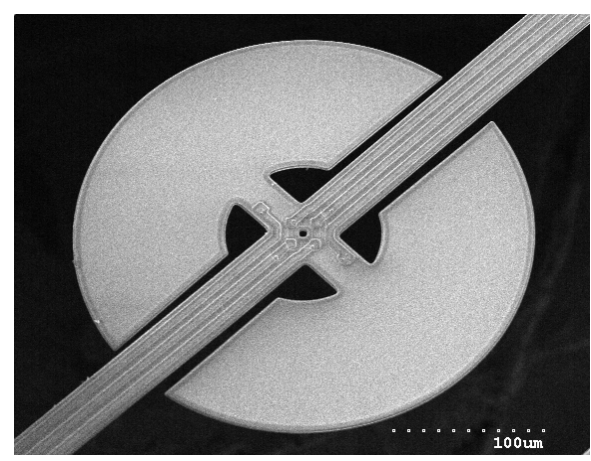

Figure 3: SEM photograph of silicon-based disk-type resonator with electrothermal excitation and piezoresistive detection elements. 
In the experiment, the temperature of the microresonator is varied to introduce a Q-factor change. A custom-made printed circuit board includes the feedback circuitry shown in Fig. 1. A disk-type resonator [10] with on-chip electrothermal excitation and piezoresistive detection elements (see Fig. 3) is placed inside an ESPEC SH-241 environmental test chamber. A LabView program controls the switch position shown in Fig. 1 via a National Instruments DAQ card and reads the chamber temperature and resonance frequency of the resonator.

\section{RESULTS}

During the measurements, the negative and positive stiffness modulation loops (switch position 1 and 2 in Fig. 1) are enabled for 2 seconds each, every 54 seconds (see Fig. 4). To prevent variations in resonance frequency by different static power dissipations with enabled and disabled modulation loop, the static power dissipation produced by the electrothermal excitation elements is kept constant during the experiment.

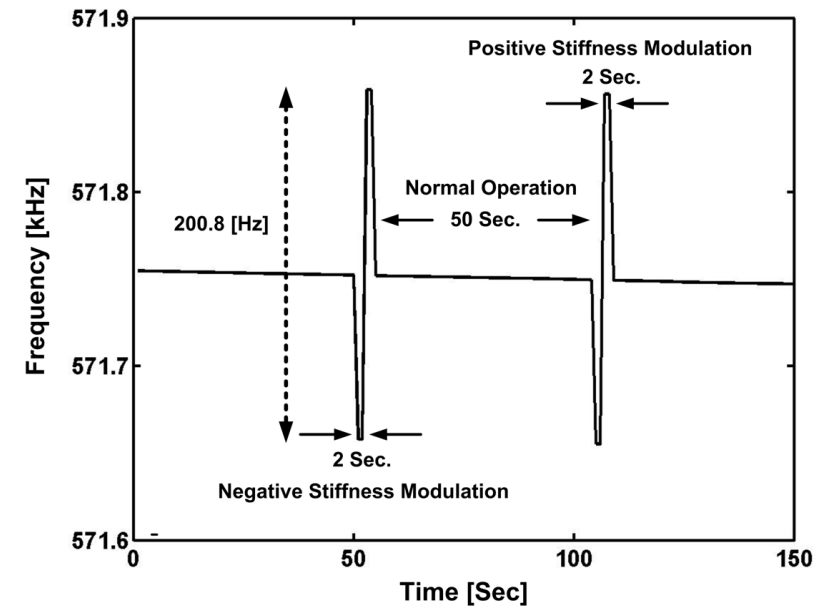

Figure 4: Resonance frequency of disk-type resonator with periodically enabled positive/negative stiffness modulation at a temperature of $\approx 10^{\circ} \mathrm{C}$.

The disk-type resonator with a $571.8 \mathrm{kHz}$ resonance frequency showed a frequency change $\left(f_{\text {pos }}-f_{\text {neg }}\right)$ of approximately $200.8 \mathrm{~Hz}$ at $10^{\circ} \mathrm{C}$ by the enabled modulation loop (see Fig. 4). As the ambient temperature increases, the frequency difference $f_{\text {pos }}-f_{\text {neg }}$ also increases (see Fig. 5), indicating that the Q-factor of the resonator is inversely proportional to the temperature. The extracted Q-factor from Eq. (8) is plotted in Fig. 6 as solid lines when the disk resonator is subjected to temperature variations between $-20^{\circ} \mathrm{C}$ and $40^{\circ} \mathrm{C}$. The Q-factors extracted from open-loop transfer characteristics of the disk resonator are plotted in Fig. 6 (symbols) for comparison.

A slight discrepancy between closed-loop and open-loop Q-factor values is observed in Fig. 6. This difference is believed to be caused by experimental errors in extracting the Q-factor of the disk resonator from open-loop measurements. The open-loop Q-factors of the disk resonator were measured only twice at the same temperature and show considerable variations (see Fig. 6). On the contrary, Q-factors estimated using the proposed stiffness modulation showed quite good repeatability and small variations over the temperature range from $-20 \sim 40^{\circ} \mathrm{C}$. Furthermore, a slight slope change in the temperature dependence of the Q-factors is noticeable around $12^{\circ} \mathrm{C}$; the origin of this effect is not fully understood yet and requires further investigation.

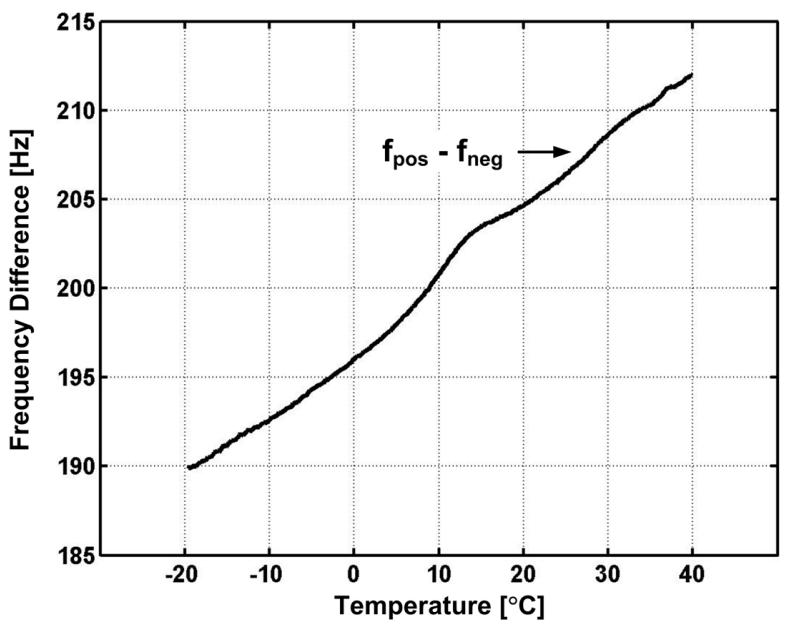

Figure 5: Frequency difference $f_{\text {pos }}-f_{\text {neg }}$ between resonator with positive and negative stiffness modulation as a function of temperature.

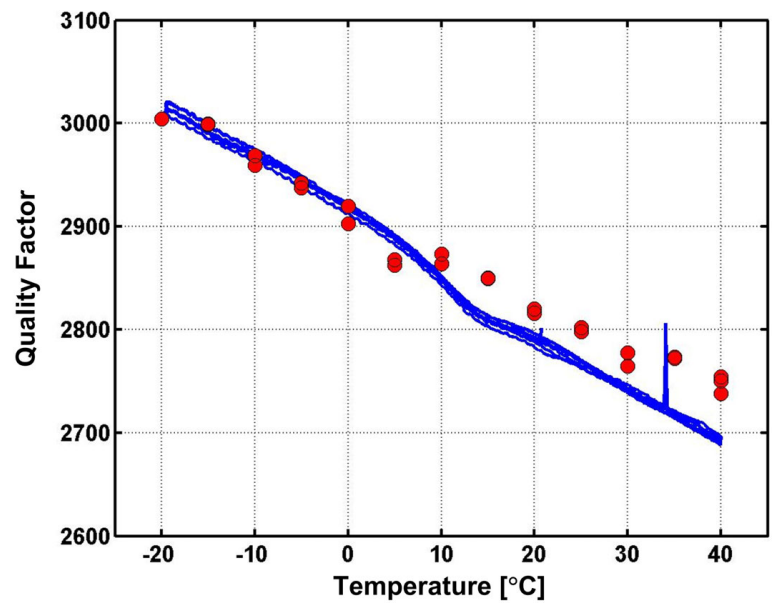

Figure 6: Comparison of quality factor extracted from Eq. (8) (solid lines) and from 3-dB bandwidth of the open-loop amplitude transfer characteristic (symbols) as a function of ambient temperature.

As a possible application of the proposed Q-factor extraction method, the extracted Q-factor change is used for temperature compensation of the microresonator using the almost linear relationship between Q-factor and temperature (see Fig. 6). The relation between temperature and Q-factor is first determined during an initial calibration step and then used to extract the temperature-induced frequency drift of the disk-type resonator. Fig. 7 shows the resonance frequency of the disk-type resonator as a function of temperature before and after temperature compensation. In this experiment, the resonator temperature is varied from $30^{\circ} \mathrm{C}$ to $60^{\circ} \mathrm{C}$. The disk-type resonator showed relative frequency changes around $1000 \mathrm{ppm}$ over the $30^{\circ} \mathrm{C}$ temperature range, while the temperature compensated frequency change was less than $50 \mathrm{ppm}$. In other words, the temperature coefficient of the resonance frequency is reduced from 33 to less than $2 \mathrm{ppm} /{ }^{\circ} \mathrm{C}$. 


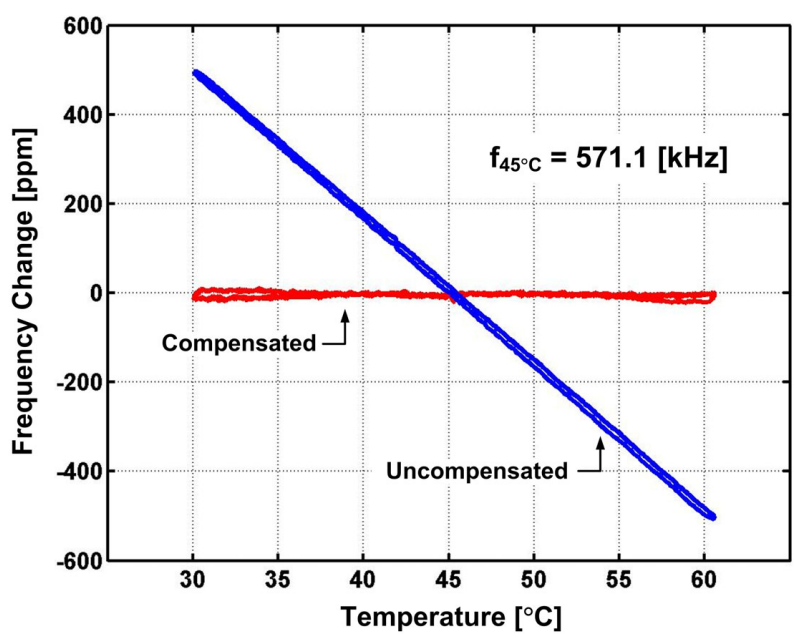

Figure 7: Relative resonance frequency change of disk-type microresonator as a function of temperature without temperature compensation (blue line) and with temperature compensation based on the Q-factor (red line); the reference frequency is $571.1 \mathrm{kHz}$ at $45^{\circ} \mathrm{C}$.

\section{DISCUSSION}

As mentioned earlier, a similar controlled-stiffness-modulation method has been used to extract the relative stiffness change of a resonator [7]. By combining the method presented in [7] and the Q-factor estimation method described in this paper, the relative stiffness change and the relative Q-factor change of a resonator can be estimated at the same time without disturbing the oscillation of a resonator at its mechanical resonance frequency. Once the relative stiffness, Q-factor and frequency changes are extracted, the changes of the remaining mechanical properties $(m, b)$ of the resonator can be estimated using:

$$
\begin{gathered}
\frac{\Delta m}{m}=\frac{\Delta k}{k}-2 \frac{\Delta \omega}{\omega} \\
\frac{\Delta b}{b}=\frac{\Delta k}{k}-\frac{\Delta Q}{Q}-\frac{\Delta \omega}{\omega}
\end{gathered}
$$

Thus, potentially all system constants of a resonator can be monitored using the proposed Q-factor tracking method in combination with the approach to extract stiffness changes [7].

\section{SUMMARY}

A method for extracting and tracking the Q-factor of a resonator during closed-loop operation at its mechanical resonance frequency is proposed and verified experimentally. The proposed method solely requires frequency measurements and can be implemented easily and cost effectively using a simple electronic feedback loop, thus avoiding expensive equipment for Q-factor measurement. By combining the Q-factor tracking method with a method for extracting relative stiffness changes, all system parameters of a resonator can potentially be monitored.

\section{ACKNOWLEDGEMENTS}

The authors would like to thank the staff of the Georgia Tech Microelectronics Research Center for their help. This work has been funded in part by the National Science Foundation under award 0601467.

\section{REFERENCES}

[1] M. K. Jain, S. Schmidt, and C. A. Grimes, "Magneto-Acoustic Sensors for Measurement of Liquid Temperature, Viscosity and Density," Applied Acoustics, vol. 62, pp. 1001-1011, 2001.

[2] M. A. Hopcroft, M. Agarwal, K. K. Park, B. Kim, C. M. Jha, R. N. Candler, G. Yama, B. Murmann, and T. W. Kenny, "Temperature Compensation of a MEMS Resonator Using Quality Factor as a Thermometer," Proc. IEEE Conf. Micro Electro Mechanical Systems (MEMS 2006), pp. 222-225, 2006.

[3] S. J. Martin, R. W. Cernosek, and J. J. Spates, "Sensing Liquid Properties with Shear-Mode Resonator Sensors," Proc. Transducers '95, vol. 2, pp. 712-715, 1995.

[4] R. Thalhammer, S. Braun, B. Devcic-Kuhar, M. Groschl, F. Trampler, E. Benes, H. Nowotny, and P. Kostal, "Viscosity Sensor Utilizing a Piezoelectric Thickness Shear Sandwich Resonator," IEEE Trans. Ultrasonics, Ferroelectrics and Frequency Control, vol. 45, pp. 1331-40, 1998.

[5] S. Bianco, M. Cocuzza, S. Ferrero, E. Giuri, G. Piacenza, C. F. Pirri, A. Ricci, L. Scaltrito, D. Bich, A. Merialdo, P. Schina, and R. Correale, "Silicon Resonant Microcantilevers for Absolute Pressure Measurement," J. Vacuum Science Technology B, vol. 24, pp. 1803-9, 2006.

[6] R. Sunier, T. Vancura, Y. Li, K.-U. Kirstein, H. Baltes, O. Brand, "Resonant Magnetic Field Sensor with Frequency Output," J. Microelectromechanical Systems, vol. 15, pp. 1098-1107, 2006.

[7] J.H. Seo, K.S. Demirci, A. Byun, S. Truax, O. Brand, "Novel Temperature Compensation Scheme for Microresonators Based on Controlled Stiffness Modulation," Proc. Transducers '07, pp. 2457-2460, 2007.

[8] J. H. Seo, "Silicon-Based Resonant Microsensor Platform for Chemical and Biological Applications," Ph.D. Thesis, Georgia Institute of Technology, 2007.

[9] K. Ogata, Modern Control Engineering, 4th ed., Prentice Hall, 2002.

[10] J.H. Seo, O. Brand, "High-Q Factor Resonant Sensor Platform for Chemical and Biological Applications," Proc. Transducers ‘05, pp. 593-596, 2005. 